\title{
Leer para ganar: el juego como dispositivo didáctico en la educación superior
}

\section{Read to win: games as a teaching device in higher education}

\author{
Carolina Clerici ${ }^{1}$ \\ María Consuelo Eckerdt ${ }^{2}$ \\ Elisa Fernanda Naef ${ }^{3}$
}

\begin{abstract}
Resumen
La lectura de textos extensos y complejos es uno de los mayores obstáculos que debe sortear el estudiante universitario, lo que se suma a la falta de interés y de tiempo para realizar lecturas sostenidas clase a clase. Esta situación evidencia la necesidad de repensar nuestras propuestas de enseñanza. Este estudio cualitativo-inductivo buscó analizar la implementación de juegos competitivos con contenidos de lecturas de cátedra obligatorias. Se analizaron las notas de la docente que implementó el dispositivo y las entrevistas a los estudiantes participantes de las actividades. Se generaron categorías de análisis mediante la utilización del método comparativo constante. Los entrevistados destacaron la motivación para leer de manera sostenida y comprensiva y valoraron el juego como instancia de aprendizaje. Es posible concluir que el juego contribuye a resolver la falta de interés de los estudiantes en la lectura de textos académicos: leen para ganar y esa lectura contribuye a la comprensión de los textos.
\end{abstract}

\footnotetext{
1 Magíster en Procesos Educativos mediados por Tecnologías. Diplomada Superior y Especialista en Ciencias Sociales, mención Lectura, Escritura y Educación. Licenciada en Educación. Profesora de Enseñanza Superior en Educación. Docente de la Universidad Nacional de Entre Ríos. Gualeguaychú, Entre Ríos, Argentina. Correo electrónico: carolina.clerici@uner.edu.ar.

${ }^{2}$ Licenciada en Nutrición. Docente de la Universidad Nacional de Entre Ríos. Paraná, Entre Ríos, Argentina. Correo electrónico: maria.eckerdt@uner.edu.ar.

${ }^{3}$ Especialista en Metodología de la Investigación. Licenciada en Nutrición. Docente de la Universidad de Concepción del Uruguay (Centro Regional Gualeguaychú). Becaria doctoral CONICET. Gualeguaychú, Entre Ríos, Argentina. Correo electrónico: elisafnaef@hotmail.com.
}

Diälogas Pedagágicas - ISSN en línea: 2524-9274.

Año XIX, No 37, abril-septiembre 2021. Pág. 1-13. DOI: http://dx.doi.org/10.22529/dp.2021.19(37)01 Recibido: 21-05-2020 / Aprobado: 26-02-2021. 
Palabras clave: didáctica, enseñanza superior, juego, lectura.

\begin{abstract}
Reading long and complex texts is one of the biggest obstacles that university students must overcome, together with the lack of interest and time to carry out sustained readings class after class. This situation shows the need to rethink our teaching approach. This qualitative-inductive study sought the analysis of the implementation of competitive games with content of compulsory academic readings. The notes of the teacher who implemented the teaching device and the interviews with the students participating in the activities were analyzed. Categories of analysis were generated using the constant comparative method. The interviewees highlighted the motivation to read in a sustained and comprehensive way and considered the games as an instance of learning. It is possible to conclude that games contribute to solve the students' lack of interest in reading academic texts: they read to win and reading contributes to understanding the texts.
\end{abstract}

Keywords: didactics, higher education, play, reading.

\title{
1. INTRODUCCIÓN
}

La lectura es una práctica frecuente en la universidad y es uno de los mayores obstáculos que enfrenta el estudiante que ingresa a la educación superior, especialmente, el hábito de leer textos extensos y complejos que, en la mayoría de los casos, es la primera vez que debe abordar. Según la UNESCO (2017), la mayoría de los latinoamericanos culminan la educación secundaria sin saber leer bien. En la universidad, la lectura no solo es más extensiva que en la escuela secundaria, sino que -también- es más intensiva; la densidad y la complejidad de los textos exigen al estudiante un mayor rigor y profundidad analítica (Arnoux, Di Stefano y Pereira, 2002; Carlino, 2003b, 2020a; Clerici, Monteverde y Fernández, 2015).

Es posible reconocer tres grandes perspectivas sobre la lectura como proceso (Roig, 2002): una que la concibe como una decodificación lineal, otra que la entiende como un proceso inferencial en el que los conocimientos previos del lector posibilitan su interacción con las características del texto y otra que la define como la producción de un texto nuevo construido por el sujeto y su contexto. Esta investigación se apoya en estas últimas: la lectura como inferencia y como construcción.

De acuerdo con lo anterior, leer -y comprender lo que se lee- implica interpretar signos y poner en juego operaciones mentales complejas; es decir que la lectura, más que un simple acto mecánico en el que se decodifican signos en forma lineal, es -principalmente- una instancia de razonamiento que nos permite interpretar un mensaje escrito. Esta práctica involucra una serie de subprocesos que el lector va realizando a medida que avanza en el texto: "la recuperación de información previa sobre el tema, la formulación de hipótesis acerca de lo que va a leer, la jerarquización de información, el procesamiento de los nuevos datos y su puesta en relación con los ya almacenados" (Arnoux et al, 2002, p. 8).

Para procesar la información, el lector realiza diversas tareas que le permiten obtener el significado global de lo que lee $y$, así, comprenderlo. En términos generales, 
se trata de reconocer las características del texto, establecer relaciones y ser capaz de expresar lo leído con sus propias palabras.

Quienes estudian la relación entre la estructura del texto y los conocimientos del lector cuentan con evidencia que permite afirmar que dicha estructura influye fuertemente en la comprensión cuando el tema les es poco familiar, mientras que pasan a ser casi irrelevantes cuando se domina ampliamente el tema tratado (McNamara, 2001; McNamara, Kintsch, Songer y Kintsch, 1996).

Además de la relación de los nuevos contenidos que presenta el texto con algún aspecto existente y relevante de la estructura cognoscitiva del alumno, es indispensable que el lector se comprometa a interaccionar significativamente con lo que lee (Ausubel, Novak y Hanesian, 1983).

Ahora bien, tanto la aptitud como la actitud en el momento de leer marcan una diferencia fundamental en la calidad de las lecturas realizadas. El lector menos hábil lee "a medias", desde una dimensión local del texto, lee palabras y frases y no desde la coherencia global (van Dijk, 1982). La lectura se realiza "sin una actitud dispuesta hacia la interpelación del texto, hacia su interrogación y su réplica, lo que supone una incomunicación con el texto" (Jurado, 2009, p. 131). Además, esto se agrava si se considera la dificultad que tienen los estudiantes de leer clase a clase (Carlino, 2020b).

Por lo tanto, si convenimos que la lectura compromete interactivamente al lector y al texto en un contexto determinado, podemos afirmar que las dificultades que se producen durante la comprensión se asocian a tres aspectos fundamentales: en primer lugar, a las características propias del texto; luego, a las competencias del lector, su bagaje cultural y sus experiencias, su conocimiento del lenguaje y de los textos y, además, se asocian a sus estados metacognitivos y afectivos y a la finalidad de la lectura. Es decir que la falta de interés en los textos académicos y la escasez de tiempo para realizar lecturas regulares y sostenidas clase a clase resultan factores que condicionan la comprensión.

A esto se suma que, como sostiene Prensky (2001, 2011), los jóvenes de hoy no pueden aprender como los de ayer, porque son diferentes sus cerebros y su cultura. Las instituciones educativas deben incorporar formatos de enseñanza basados en el ocio y el entretenimiento. Resulta evidente que nuestros estudiantes piensan y procesan la información de modo significativamente distinto a sus predecesores. Como afirma este autor, prefieren los juegos al trabajo "serio".

El juego aporta una cuota de azar que genera un interés derivado de la incertidumbre y de la imposibilidad de predecir qué va a suceder. En esta investigación, se entiende por lúdico lo relacionado con el juego en su acepción de entretenimiento y recreación (Real Academia Española [RAE], 2014), es decir, como un ejercicio placentero que permite liberarse de tensiones y de las reglas impuestas por la cultura. Huizinga (1954, 2007) describe el juego como una acción o una ocupación libre "que se desarrolla dentro de unos límites temporales y espaciales determinados, según reglas absolutamente obligatorias, aunque libremente aceptadas, acción que tiene su fin en sí misma y acompañada de un sentimiento de tensión y alegría" (pp. 45-46).

En la educación formal, el juego y las actividades lúdicas son reconocidas y valoradas como recurso metodológico, particularmente en los niveles inicial y primario (Savazzini, 2014). También es habitual el uso de estas dinámicas en la educación secundaria y son muchas las propuestas de formación y entrenamiento profesional 
(como coaching) que incluyen lo lúdico como recurso primordial. Sin embargo, esto no se da con frecuencia en la educación superior.

En el imaginario social, es frecuente que el juego se asocie a la pérdida de tiempo y se lo abandone para dar paso a otro tipo de actividades "más serias". En este sentido:

Imaginar momentos relacionados con lo lúdico en un aula universitaria se podría tornar polémico, cuando en realidad la instancia de juego, en cualquier ámbito educativo, nos abre las puertas al intercambio de experiencias y de conocimientos, en un ambiente de disfrute. (Rubio, 2012, p. 151)

Además, las actividades lúdicas contribuyen a dejar de lado tensiones, prejuicios e individualismos y se convierte en un medio de expresión por excelencia. Más recientemente, se ha asociado el juego a la gamificación, la ludificación y el aprendizaje basado en juegos (Game-Based Learning). Según Abt (1970), los serious games [juegos serios] son juegos con fines educativos explícitos que están cuidadosamente pensados para no ser destinados únicamente a la diversión.

El juego, en la universidad, permite la participación activa de los estudiantes (Álvarez, 2010; Clerici, 2012) y promueve "la aparición de mayor creatividad, flexibilidad, distensión grupal, creación de buen clima de trabajo, cohesión grupal, y aprendizaje significativo de contenidos que estén incluidos en el dispositivo lúdico" (Savazzini, 2014 , p. 190). Jugar implica una acción y en tanto tal "es una de las herramientas más efectivas para promover el aprendizaje y transferir el conocimiento gracias a su capacidad de simular la realidad ofreciendo un escenario para cometer errores y aprender de ellos en la práctica" (Álvarez, 2010, p. 1).

A las dificultades antes mencionadas para la comprensión, a saber, las características propias del texto, las competencias del lector y sus estados metacognitivos y afectivos, se suma el hecho de que nuestros alumnos aprenden de un modo diferente. Esto nos incita a afinar nuestras metodologías, que deberán considerar no solo los contenidos disciplinares y la forma de presentarlos, sino también las características particulares de los estudiantes destinatarios de nuestra enseñanza. Se trata de reinventar la clase (Maggio, 2018).

Asimismo, todo acto de enseñanza busca producir cambios o algún tipo de transformación en aquellos hacia quienes se dirige la acción pedagógica (Fenstermacher, 1989). Esta pretensión suele ponerse en marcha según un proyecto que orienta las prácticas que se emprenden. La enseñanza como actividad intencional es también un proceso de producción, de creación, de innovación y, además, podría incluir instancias de prueba y experimentación (distinta a la que la concibe como mera "aplicación"). Entendemos que una buena práctica de enseñanza se basa en la configuración de intencionalidades definidas y explícitas. Es aquella en la que el docente apela a recursos nuevos o existentes, encuentra un sentido para ese hacer, lo lleva a la práctica, recupera lo accionado y piensa en mejorar futuras prácticas.

No existen recetas únicas para pensar dispositivos a la hora de enseñar en la universidad ni para lograr la originalidad de las propuestas y escenarios de enseñanza que diseñamos para evitar la rutina en el aula. Esto desafía la creatividad, la innovación, y forma parte del complejo y maravilloso oficio de enseñar. Esta situación evidencia la necesidad de pensar dispositivos que enriquezcan las clases con modalidades que puedan generar en los estudiantes una nueva "vuelta" sobre los contenidos que se abordan en espacios de enseñanza. 
Consideramos que el dispositivo analizado en esta investigación intenta recrear situaciones de "enseñanza poderosa", en línea con Maggio (2012) cuando se refiere a una enseñanza que "explota" en el marco de la clase y en el recuerdo de aquellas prácticas que marcan. Por ello, el objetivo de este estudio fue describir un dispositivo didáctico basado en juegos competitivos con contenidos de lecturas de cátedra obligatorias, conocer la mirada de la docente a cargo de la propuesta y de los estudiantes que participaron en ella.

\section{METODOLOGÍA}

Se trata de un estudio cualitativo que busca comprender el impacto de un dispositivo didáctico basado en juegos competitivos con contenidos de lecturas de cátedra obligatorias. El estudio analiza datos recolectados en tres poblaciones de instituciones de educación superior: (1) estudiantes del Taller de Investigación Educativa de tercer año del Profesorado en Economía, (2) docentes y licenciados que cursaron Lingüística y Comunicación Humana en el Ciclo Complementario del Profesorado de Enseñanza Superior y (3) docentes que cursaron Procesos Educativos y Problemáticas Situadas en el ciclo de la Licenciatura en Pedagogía.

Por tratarse de un fenómeno que no ha sido abordado en profundidad, se propuso un estudio cualitativo exploratorio-descriptivo, desde una lógica inductiva, que buscó la generación de categorías de análisis que permitiesen conocer el uso de actividades lúdicas en la educación superior. Se trata de un diseño a través del cual se analizó la información empírica y se generaron categorías teóricas para conocer el fenómeno bajo estudio.

Se analizaron las notas de campo que la docente a cargo de la aplicación del dispositivo fue registrando clase a clase para conocer el sentido del uso de este tipo de actividades y la valoración realizada desde su perspectiva. Se indagó sobre el motivo por el cual se introdujeron este tipo de actividades, el modo de implementación, las expectativas con las que se realizaron, la valoración de dichas actividades desde su propia perspectiva y los factores que han facilitado u obstaculizado su puesta en marcha.

Además, se realizaron entrevistas a los estudiantes que habían participado de estas actividades con el propósito de conocer su mirada en relación con las intenciones y expectativas del docente. En esta ocasión, se indagó sobre el sentido de las actividades lúdicas, la valoración desde su punto de vista y la posibilidad de utilizar juegos en su propia práctica, considerando especialmente que las tres poblaciones de estudio estaban cursando una carrera de formación docente.

Para el tratamiento de los datos, se utilizó el método comparativo constante (Glaser y Strauss, 1967), lo que implicó que, a medida que se avanzó en el trabajo de campo, se codificó y se analizó la información empírica simultáneamente y se desarroIlaron conceptos a partir de la comparación continua entre la información empírica y la teoría en una relación dialéctica (Sirvent y De Angelis, 2011).

Esto ha permitido dar una vuelta a la teoría a partir de la información recolectada con el propósito de realizar un aporte al conocimiento en esta área de estudio. Este diseño permitió explicar -en un nivel conceptual- una acción, en este caso, el uso de actividades lúdicas en la educación superior desde los datos empíricos obtenidos, más 
que desde estudios previos, a la vez que contribuyó a comprender el fenómeno en su complejidad.

\section{RESULTADOS Y DISCUSIÓN}

En este artículo, se describen los primeros resultados de una investigación de mayor envergadura que buscó describir un dispositivo didáctico basado en juegos competitivos con contenidos de lecturas de cátedra obligatorias, conocer la mirada de la docente a cargo de la propuesta y de los estudiantes que participaron en ella. En este apartado, se incluyen las categorías que surgieron con más fuerza de los datos empíricos y que se asocian particularmente a aspectos como el dispositivo propiamente dicho, la motivación para leer, los conocimientos previos y los aprendizajes inesperados. Para salvaguardar la identidad de los participantes, se citan sus palabras utilizando la letra E seguida del número de entrevista.

\subsection{El juego como dispositivo didáctico}

Se implementó un dispositivo pensado para hacer frente a la falta de motivación de los estudiantes para la lectura de textos de la cátedra. La propuesta general puede describirse como una seguidilla de juegos para los que tenían que leer si querían ganar. Se solicitaba la lectura de un texto, un capítulo o un artículo, de una clase para la siguiente $y$, en esa clase, se proponía un juego competitivo basado en el contenido del texto asignado para la lectura. Finalmente, se realizaba un cierre del tema a cargo de la docente o de uno de los estudiantes. La Figura 1 ilustra el dispositivo.

Figura 1

Esquema general de las clases basadas en juegos

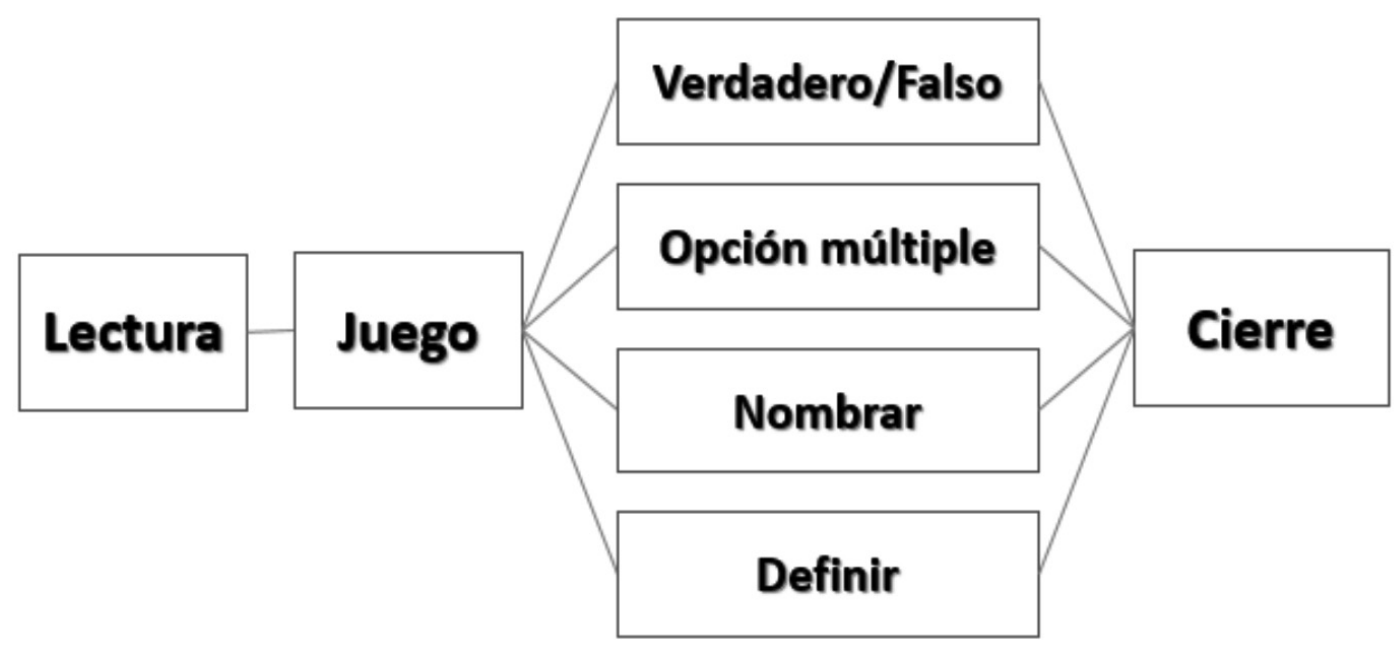

Fuente: Elaboración propia.

Cada clase iniciaba con la conformación de grupos que disponían de unos minutos para recordar la lectura y compartir sus notas antes de comenzar a jugar. Si bien cada 
juego era diferente y tenía sus propias características y reglas, solo se trataba de cuatro formatos según la respuesta que se esperaba de los estudiantes: verdadero-falso, opción múltiple, respuesta cerrada y respuesta abierta. Respuesta cerrada se refería a aquellos en los que, por ejemplo, se leía una definición y ellos debían nombrar el concepto definido. Los juegos de respuesta abierta fueron aquellos en los que se realizaba una pregunta a desarrollar en forma breve o se pedía la definición o explicación de algún concepto. Algunos de los juegos analizados fueron iguales o adaptados de juegos conocidos como, por ejemplo, Carrera de mente, "dígalo con mímica" y "batalla naval"; otros fueron creados por la docente, como "los náufragos", "el pica-pica", "el semáforo", "la rapisilla", "apuesta tu respuesta", "casino temático", entre otros.

\subsection{El juego funciona si tu dispositivo es adecuado}

En las notas de la docente, se registró que existía la motivación de leer semana a semana para estar preparados para el juego: "Ellos leían porque querían ganar" escribió la docente. Observó que esta actitud se sostenía en el tiempo y "cada vez eran más los estudiantes que llegaban a clase con los textos trabajados, subrayados y sabidos". Destacó que la inclusión del juego en el aula, particularmente en la universidad, puede resultar un desacierto si no impulsa la lectura y el tratamiento de los contenidos y si no se sostiene en el tiempo.

Los estudiantes reconocieron que concurrir a clase sin haber leído implicaba un obstáculo para el desarrollo del juego. En los siguientes fragmentos, se vislumbra la importancia de que el dispositivo sea adecuado, es decir, que los estudiantes lean antes de cada clase:

Sirven, en forma positiva, si hubo una lectura previa de las mismas. (E8)

En algunos casos era tedioso encontrar en lectura rápida las respuestas y variaba según el grupo en el que te encontrabas. Si alguien había leído era fácil, pero cuando el tiempo, tirano por cierto, no nos había permitido leer a conciencia algún autor, dificultaba la actividad y la misma se volvía por momentos tediosa. (E7)

Requiere un compromiso por parte de los alumnos de leer previamente el material de estudio porque si no, no se puede participar muy activamente de las actividades de estos juegos. (E10)

Estos testimonios sugieren que la introducción del juego en el aula tiene sentido si se integra con responsabilidad y compromiso. No se trata de introducir actividades lúdicas para entretener a los estudiantes, sino de motivarlos para el abordaje de textos complejos, "que tengas ganas de leer", como registró la docente.

Además, si bien todos los artefactos de la modernidad pueden ser propicios para el aprendizaje, debemos orientar su uso a fin de evitar aspectos perjudiciales, optimizando el empleo de esos instrumentos y neutralizando sus debilidades (Corchuelo-Rodríguez, 2018; Rubio, 2012; Savazzini, 2014). Al implementar el juego en la universidad, es necesario cuidar el diseño, planeación e implementación de la estrategia, dado que de esas mecánicas depende su éxito o fracaso (Corchuelo-Rodríguez, 2018). 


\subsection{Motivación para leer (y ganar)}

Los estudiantes coincidieron en destacar la motivación que generó el dispositivo para leer para cada clase, particularmente, para realizar una lectura en forma comprensiva. Y también manifestaron lo ameno que resultaba "llevar las lecturas al día". Además, destacaron el dispositivo como algo diferente, que sale de la rutina y que genera motivación. Los siguientes testimonios dan cuenta de que el deseo de ganar fue tal que leían para cada encuentro y que era impensado ir a clase sin haber realizado la lectura correspondiente. En este sentido, el juego involucra el conjunto de la personalidad y potencia el aprendizaje significativo (Ausubel, Novak y Hanesian, 1983):

Las actividades lúdicas fueron muy beneficiosas para tenernos enganchados en cada tema y con la materia en general, el hecho de que en cada clase se generaba una "competencia" con el contenido nuevo a desarrollar hacía impensado concurrir sin leer, al menos algo, del material acordado. (E3)

Este tipo de actividades sirven para el desarrollo del proceso de aprendizaje porque aquí entra en juego la motivación de los estudiantes, como el objetivo por parte de los chicos siempre será ganar, harán lo imposible para conseguirlo, incluso estudiar. (E11)

Además, la calidad de la lectura también se vio afectada por el deseo de ganar. Expresaron que realizaban lecturas a conciencia y "en serio", motivados por la competencia:

Más que las actividades lúdicas en sí mismas, la necesidad de competir me ayudó a, sí o sí, tener que hacer una lectura a conciencia del material bibliográfico. (E4)

Las actividades lúdicas me sirvieron para estudiar más, leer en serio antes de cada clase. (E6)

La motivación para leer regularmente y la toma de conciencia de la necesidad de hacer lecturas comprensivas se presentan en las palabras de los entrevistados como una de las fortalezas más directamente asociadas a los objetivos expresados por la docente. En palabras de Perrenoud (1998), "para que una actividad sea generadora de aprendizaje, es necesario que la situación desafíe al sujeto, que a este le apetezca enfrentarse a ella, y que todo ello esté dentro de sus posibilidades, a costa de un aprendizaje nuevo" (p. 14). Los entrevistados refirieron reiteradamente al desafío que implicó tener que leer y al valor de esas lecturas previas para el juego y para el aprendizaje.

\subsection{Aprendieron porque habían leído (casi sin darse cuenta)}

Uno de los aspectos que destacaron los estudiantes fue que la lectura previa permitió relacionar contenidos y aprender. En este sentido, se evidenciaría un proceso de aprendizaje significativo y no memorístico. Esto se ilustra en palabras de los entrevistados:

Una manera de integrar conocimientos, de fomentar la comprensión y la memoria, ya que a través de los juegos pusimos en práctica estas habilidades, y por mi experiencia comprobé que recordaba al finalizar el año conceptos que 
habíamos estudiado al principio, que tal vez estudiándolos de memoria no lo lograría. (E13)

La verdad, me sorprendieron en el primer momento las actividades lúdicas, me costó al inicio aplicarme leyendo los textos, pero después me di cuenta de que aprendía muchísimo y entonces leía y me preparaba para jugar. (E9)

Los estudiantes expresaron que pudieron visualizar y tomar conciencia de lo que habían aprendido a través de la lectura y los juegos porque eran capaces de recordar los contenidos abordados al finalizar la asignatura. Se observa un proceso gradual y no asociado al esfuerzo por memorizar.

\subsection{Más allá del juego}

Además de los aspectos destacados anteriormente, que de algún modo son parte del dispositivo pensado por la docente, los estudiantes refirieron aprendizajes que fueron más allá del juego. En general, se relacionaron con la creatividad, el trabajo en equipo, la expresión oral y la posibilidad de aprender del error.

Aprendí otra manera de aprender un tema mediante un juego. (E1)

Este fue el primer año que experimenté en esta materia actividades lúdicas, si bien al principio no me sentí muy a gusto, pero creo que todo fue porque no estaba acostumbrada y el prejuicio que uno tiene en pensar "con un 'juego' no voy a aprender". Me di cuenta de que jugando se aprende un montón y creo que aún más de lo normal, ya que, como dije anteriormente, genera emociones y, ivaya!, qué emociones, en mí generó alegría, sobre todo en querer leer para saber qué juego habrá, si no, creo que, a falta de tiempos por parciales y demás, no me hubiese sentido motivada a leer. (E12)

Aprendés casi sin darte cuenta, se socializa mucho el conocimiento, si te equivocás, lo hacés en este momento y no en otro; y se integra el grupo; incluso ayudan a generar confianza. (E5)

Los estudiantes señalaron el hecho de que el juego posibilita conocer otros modos de aprender, diferentes de la clase expositiva tradicional. "Se trata de desdramatizar lo dramático con el firme propósito de aprender, sin sentir los riesgos que conlleva el error sino de reconocerlos como una estrategia de aprendizaje válido para cualquier tiempo y circunstancia" (Lion y Perosi, 2017, p. 46).

Además, el aprendizaje al que refieren como resultado de la socialización del conocimiento indica que el juego promueve la generación de una zona de desarrollo próximo en tanto el estudiante resuelve situaciones que exceden su posibilidad de respuesta individual, pero que logra resolver con compañeros más capaces (Vigotsky, 1988 , p. 133).

\section{CONCLUSIÓN Y DISCUSIóN}

Con esta investigación, se ha buscado analizar un dispositivo didáctico basado en juegos competitivos con contenidos de lecturas de cátedra obligatorias. Aunque los resultados son sugerentes, las características del diseño y el tamaño y el tipo de muestra solo sirven para describir un fenómeno particular y no para generalizar. Te- 
niendo en cuenta estas limitaciones, es posible observar que los resultados, en diálogo con el marco teórico en el que se sustenta, evidencian la pertinencia y el valor de la propuesta tanto para la docente a cargo como para los estudiantes que participaron en ella. Se destaca particularmente el hecho de que los estudiantes, motivados por el juego y la competencia, leen para cada clase, leen a conciencia y aprenden, porque la lectura actúa como conocimiento previo para un aprendizaje significativo y, además, reconocen otros aprendizajes como, por ejemplo, aprender otros modos de aprender.

La riqueza que abre el juego dentro del aula justifica darle espacio en escenarios académicos universitarios, favorecedores de motivación, dinámicas de grupo, atención crítica reflexiva y apropiación de aprendizajes significativos. Sostenemos que, si el planteamiento es adecuado y responde a objetivos concretos, las actividades lúdicas se convierten en estrategias de enseñanza y aprendizaje justificadas en la clase universitaria (Clerici, 2012; Corchuelo-Rodríguez, 2018; Rubio, 2012).

Además del valor propio del dispositivo didáctico analizado en esta investigación, se destaca el compromiso de los educadores con el desarrollo de habilidades para la lectura. La comprensión lectora, tal como se abordó en esta investigación, se inscribe en el contexto académico: un ámbito marcado por la exigencia comunicativa en el que circulan textos con rasgos lingüísticos, discursivos y retóricos muy particulares y que son propios de las prácticas de comunicación que se dan en ese contexto (Navarro, 2014; Parodi y Burdiles, 2015). Las prácticas discursivas en el ámbito universitario requieren de un aprendizaje específico.

En consecuencia, existe consenso para sostener que, al inicio de los estudios superiores, es un objetivo central introducir, de manera sistemática, a los estudiantes en la lectura y producción de géneros académicos orales y escritos. Si la universidad no ofrece instancias en las que los estudiantes puedan desarrollar las competencias necesarias para la etapa de formación que se proponen transitar, solamente tendrán éxito quienes por tradición familiar, por el ámbito social al que pertenecen o porque hayan sido beneficiados por una escolaridad privilegiada podrán continuar con su carrera hasta graduarse. El escaso manejo de habilidades discursivas en el contexto científico-académico excluirá a los demás de la universidad (Moyano, 2007). La lectura, entendida como sistema complejo que requiere conocimiento especializado, puede convertirse en un canal de expulsión en el sentido que le imprime Sassen (2014).

Independientemente del área disciplinar en que nos desempeñemos, la práctica de la lectura es una responsabilidad compartida (Carlino, 2003a, 2004; Cassany y Morales, 2008; Moyano, 2010; Parodi y Burdiles, 2015), como también lo es el desarrollo de competencias para leer propias de cada asignatura en particular. Se trata de aprendizajes continuos, desde la escuela primaria a la universidad, y transversales, ya que atañen a todos los campos disciplinares. Esto implica que debemos proponer la lectura en nuestra disciplina y también debemos enseñar cómo hacerlo.

Ser lector académico es el resultado de un proceso complejo que reconoce en su base otros procesos de iguales características desarrollados en etapas previas de la alfabetización. Es necesario ofrecer a los estudiantes herramientas para abordar textos académicos y científicos. La recuperación del valor de la lectura no radica solamente en el hecho de ser un medio para acceder a la información, sino como instrumento poderoso para generar y transformar conocimiento, mejorar la calidad de los aprendizajes e involucrar a los estudiantes en su propio proceso de formación. La lectura es una habilidad esencial de la que debe ocuparse la educación; es un instrumento que 
nos permite penetrar la cultura y ser penetrados por ella, es la vía de acceso al pasado codificado y al presente que no vemos con nuestra experiencia directa. La formación de lectores críticos y la construcción de la ciudadanía son propósitos centrales de las instituciones educativas. El acceso y el dominio pleno de esta práctica son un derecho y una vía para posibilitar una enseñanza más democratizadora e inclusiva.

\section{REFERENCIAS BIBLIOGRÁFICAS}

Abt, C. (1970). Serious Games. New York: Viking Press.

Álvarez, M. M. (2010). El juego en la Universidad: relato de una experiencia. Quaderns digitals: Revista de Nuevas Tecnologías y Sociedad, 62, 10. Recuperada el 16/03/2021 de: http://www. quadernsdigitals.net/datos/hemeroteca/r_1/nr_809/a_10931/10931.pdf

Arnoux, E., Di Stefano, M. \& Pereira, C. (2002). La lectura y la escritura en la universidad. Buenos Aires: Eudeba.

Ausubel, P., Novak, J. \& Hanesian, H. (1983). Psicología educativa: Un punto de vista cognitivo. México: Trillas.

Carlino, P. (2003a). Alfabetización académica: Un cambio necesario, algunas alternativas posibles. Educere, 6(20), 409-420. Recuperado el 10/03/2021 de: https://www.redalyc.org/ pdf/356/35662008.pdf.

Carlino, P. (2003b). Leer textos científicos y académicos en la educación superior: Obstáculos y bienvenida a una cultura nueva. Uni-pluri/versidad, 3(2), 17-23. Recuperado el 20/02/2021 de: https://aprendeenlinea.udea.edu.co/revistas/index.php/unip/article/viewFile/1289/11146.

Carlino, P. (2004). Escribir a través del currículum: Tres modelos para hacerlo en la universidad. Lectura y Vida. Revista Latinoamericana de Lectura, 25(1), 16-27. Recuperado el 28/02/2021 de: https://media.utp.edu.co/referencias-bibliograficas/uploads/referencias/articulo/248-escribir-a-travs-del-curriculum-tres-modelos-para-hacerlo-en-la-universidadpdf-jQyDB-articulo.pdf.

Carlino, P. (2020a). Leen pero no comprenden... Qué puede hacer la universidad para acompañar la lectura en las disciplinas. En E. Ramírez Leyva, La formación de lectores más allá del campo disciplinar (pp. 17-30). México (México): UNAM. Instituto de Investigaciones Bibliotecológicas.

Carlino, P. (2020b). Para que los universitarios lean clase a clase... (¿de quién es el problema?). En E. Ramírez Leyva, La formación de lectores más allá del campo disciplinar (113-129). México (México): UNAM. Instituto de Investigaciones Bibliotecológicas.

Cassany, D. \& Morales, O. A. (2008). Leer y escribir en la universidad: hacia la lectura y la escritura crítica de géneros científicos. Memoralia, 5(2), 69-82. Recuperado el 05/03/2021 de: http://www.saber.ula.ve/bitstream/handle/123456789/16457/leer_universidad.pdf;jsessionid =F8E48AF0E97F0C6E879F47E7E666C1B9? sequence $=1$.

Clerici, C. (2012). El juego como estrategia de enseñanza y aprendizaje en el nivel superior. Revista Diálogos Pedagógicos, 10(19), 136-140. Recuperado el 19/02/2021 de: http://revistas. bibdigital.uccor.edu.ar/index.php/dialogos/article/view/199/11.

Clerici, C., Monteverde, A. C. \& Fernández, A. (2015). Lectura, escritura y rendimiento académico en ingresantes universitarios. Ciencia, Docencia y Tecnología, 26(50), 35-70. Recuperado el 11/03/2021 de: http://pcient.uner.edu.ar/index.php/cdyt/article/view/59. 
Corchuelo-Rodríguez, C. A. (2018). Gamificación en educación superior: experiencia innovadora para motivar estudiantes y dinamizar contenidos en el aula. EDUTEC. Revista Electrónica de Tecnología Educativa, 63, 29-41. https://doi.org/10.21556/edutec.2018.63.927.

Fenstermacher, G. (1989). Tres aspectos de la filosofía de la investigación en la enseñanza. En M. Wittrock (Comp.), La investigación en la enseñanza I. Enfoques, teorías y métodos (pp. 150181). Barcelona: Paidós.

Glaser, B. \& Strauss, A. (1967). The discovery of grounded theory: strategies for qualitative research. Nueva York: Aldine Publishing Company.

Huizinga, J. (1954/2007). Homo ludens. (Trad. E. Imaz). Madrid: Alianza Editores.

Jurado, F. (2009). La evaluación de la escritura para la evaluación de la lectura. Revista Iberoamericana de Evaluación Educativa, 2(1), 131-146. Recuperado el 23/02/2021 de: https:// revistas.uam.es/index.php/riee/article/view/4573

Lion, C. \& Perosi, V. (2017). Los videojuegos serios: puentes de creatividad y expansión educativa. Anales de la Educación común, 2(2), 45-49.

Maggio, M. (2012). Enriquecer la enseñanza. Buenos Aires: Paidós.

Maggio, M. (2018). Reinventar la clase en la Universidad. Buenos Aires: Paidós.

McNamara, D. S., Kintsch, E., Songer, N. B. \& Kintsch, W. (1996). Are good texts always better? Interactions of text coherence, background knowledge, and levels of understanding in learning from text. Cognition and Instruction, 14(1), 1-43. https://doi.org/10.1207/s1532690xci1401_1.

McNamara, D. S. (2001). Reading both high-coherence and low-coherence texts: Effects of text sequence and prior knowledge. Canadian Journal of Experimental Psychology/Revue canadienne de psychologie expérimentale, 55(1), 51-62. https://doi.org/10.1037/h0087352.

Moyano, E. (2007). Enseñanza de habilidades discursivas en español en contexto pre-universitario: Una aproximación desde la LSF. Revista signos, 40(65), 573-608. http://dx.doi.org/10.4067/ S0718-09342007000300009

Moyano, E. (2010). Escritura académica a lo largo de la carrera: Un programa institucional. Revista Signos, 43(74), 465-488. http://dx.doi.org/10.4067/S0718-09342010000500004

Navarro, F. (Coord.) (2014). Manual de escritura para carreras de las humanidades. Buenos Aires: Universidad de Buenos Aires.

Parodi, G. \& Burdiles, G. (Eds.). (2015). Leer y escribir en contextos académicos y profesionales: Géneros, Corpus y Métodos. Santiago de Chile: Ariel.

Perrenoud, P. (1998) ¿A dónde van las pedagogías diferenciadas? Hacia la individualización del currículo y de los itinerarios formativos. Universidad de Ginebra. Facultad de Psicología y de Ciencias de la Educación. Educar, 22-23, 11-34.

Prensky, M. (2001). Digital Natives, Digital Immigrants. On the Horizon, MCB University Press, 9(5), 1-6.

Prensky, M. (2011). From Digital Natives to Digital Wisdom. Hopeful Essays for $21^{\text {st }}$ Century Education. Recuperado el 25/01/2021 de: https://marcprensky.com/writing/Prensky-Intro_to_ From_DN_to_DW.pdf.

Real Academia Española [RAE] (2014). Diccionario de la lengua española (23a edición). Madrid: Espasa. 
Roig, H. (2002). Lectura de textos audiovisuales y prácticas educativas: el aula universitaria como contexto particular de recepción (Tesis doctoral). Universidad de Buenos Aires, Argentina. Recuperado el 26/01/2021 de: http://repositorio.filo.uba.ar/handle/filodigital/1367.

Rubio, A. (2012). El juego en la universidad. Reflexión Académica en Diseño y Comunicación, 13(19) 150-153. Recuperado el 20/12/2020 de: http://fido.palermo.edu/servicios_dyc/publicacionesdc/archivos/380_libro.pdf.

Sassen, S. (2014). Expulsions: Brutality and Complexity in the Global Economy. Cambridge, MA, USA: Harvard University Press.

Savazzini, M. (2014). Reflexiones acerca de la utilización del juego como estrategia en Educación Superior: El cuerpo docente en juego. Reflexión Académica en Diseño y Comunicación, 15(23) 189-193. Recuperado el 25/12/2020 de: https://fido.palermo.edu/servicios_dyc/publicacionesdc/archivos/484_libro.pdf.

Sirvent, M. T. \& De Angelis, S. (2011). Pedagogía de formación en investigación. Cuadernos de Educación, 9(9), 231-244. Recuperado el 31/12/2020 de: https://revistas.unc.edu.ar/index.php/ Cuadernos/article/viewFile/835/787.

UNESCO Instituto de Estadística (2017). Más de la mitad de los niños y adolescentes en el mundo no están aprendiendo. Recuperado el 01/01/2021 de: http://uis.unesco.org/sites/default/files/ documents/fs46-more-than-half-children-notlearning-2017-sp.pdf.

van Dijk, T. (1982). La ciencia del texto. Barcelona: Paidós.

Vigotsky, L. (1988). El desarrollo de los procesos psicológicos superiores. Madrid: Crítica Grijalbo. 